PROCEEDINGS OF THE

AMERICAN MATHEMATICAL SOCIETY

Volume 130, Number 8, Pages 2197-2202

S 0002-9939(02)06424-9

Article electronically published on January 31, 2002

\title{
SUR UNE QUESTION DE CAPITULATION
}

\author{
ABDELMALEK AZIZI
}

(Communicated by David E. Rohrlich)

\begin{abstract}
Let $p$ and $q$ be prime numbers such that $p \equiv 1 \bmod 8, q \equiv-1 \bmod$ 4 and $\left(\frac{p}{q}\right)=-1$. Let $d=p q, \mathbf{k}=\mathbf{Q}(\sqrt{d}, i)$, and let $\mathbf{k}_{2}^{(1)}$ be the 2-Hilbert class field of $\mathbf{k}, \mathbf{k}_{2}^{(2)}$ the 2-Hilbert class field of $\mathbf{k}_{2}^{(1)}$ and $G_{2}$ the Galois group of $\mathbf{k}_{2}^{(2)} / \mathbf{k}$. The 2-part $C_{\mathbf{k}, 2}$ of the class group of $\mathbf{k}$ is of type $(2,2)$, so $\mathbf{k}_{2}^{(1)}$ contains three extensions $\mathbf{K}_{i} / \mathbf{k}, i=1,2,3$. Our goal is to study the problem of capitulation of the 2-classes of $\mathbf{k}$ in $\mathbf{K}_{i}, i=1,2,3$, and to determine the structure of $G_{2}$.

RÉSUmÉ. Soient $p$ et $q$ deux nombres premiers tels que $p \equiv 1 \bmod 8, q \equiv$ $-1 \bmod 4$ et $\left(\frac{p}{q}\right)=-1, d=p q, i=\sqrt{-1}, \mathbf{k}=\mathbf{Q}(\sqrt{d}, i), \mathbf{k}_{2}^{(1)}$ le 2-corps de classes de Hilbert de $\mathbf{k}, \mathbf{k}_{2}^{(2)}$ le 2-corps de classes de Hilbert de $\mathbf{k}_{2}^{(1)}$ et $G_{2}$ le groupe de Galois de $\mathbf{k}_{2}^{(2)} / \mathbf{k}$. La 2-partie $C_{\mathbf{k}, 2}$ du groupe de classes de $\mathbf{k}$ est de type $(2,2)$, par suite $\mathbf{k}_{2}^{(1)}$ contient trois extensions $\mathbf{K}_{i} / \mathbf{k}, i=1,2,3$. On s'intéresse au problème de capitulation des 2-classes de $\mathbf{k}$ dans $\mathbf{K}_{i}, i=1,2,3$, et à déterminer la structure de $G_{2}$.
\end{abstract}

\section{INTRODUCTION}

Soient $\mathbf{k}$ un corps de nombres de degré fini sur $\mathbf{Q}, \mathbf{F}$ une extension non ramifiée de $\mathbf{k}$ et $p$ un nombre premier. L'extension $\mathbf{k}^{(1)}$ de $\mathbf{k}$, abélienne maximale et nonramifiée pour tous les idéaux premiers, finis et infinis, est dite corps de classes de Hilbert de $\mathbf{k}$. De même l'extension $\mathbf{k}_{p}^{(1)}$ de $\mathbf{k}$ dont le degré est une puissance de $p$, abélienne maximale et non-ramifiée pour tous les idéaux premiers, finis et infinis, est dite $p$-corps de classes de Hilbert de $\mathbf{k}$.

La recherche des idéaux de $k$ qui capitulent dans $\mathbf{F}$ (deviennent principaux dans $\mathbf{F}$ ), a été l'objet d'étude d'un grand nombre de mathématiciens. En effet, Kronecker était parmi les premiers à avoir abordé des problèmes de capitulation dans le cas des corps quadratiques imaginaires. Dans le cas où $\mathbf{F}$ est égal au corps de classes de Hilbert $\mathbf{k}^{(1)}$ de $\mathbf{k}$, D. Hilbert avait conjecturé que toutes les classes de $\mathbf{k}$ capitulent dans $\mathbf{k}^{(1)}$ (théorème de l'idéal principal). La preuve de ce dernier théorème a été réduite par $\mathrm{E}$. Artin à un problème de la théorie des groupes, et c'est $\mathrm{Ph}$. Furtwängler qui l'avait achevée. Le théorème de l'idéal principal a été généralisé de la façon suivante: Soient $\mathbf{k}_{0}$ un corps de nombres et $\mathbf{k} / \mathbf{k}_{0}$ une

Received by the editors February 23, 2001.

2000 Mathematics Subject Classification. Primary 11R37.

Key words and phrases. Groupe des unités, système fondamental d'unités, capitulation, corps de classes de Hilbert. 
extension cyclique finie. Alors toutes les classes ambiges (les classes stables par le groupe de Galois de $\mathbf{k} / \mathbf{k}_{0}$ ) de $\mathbf{k}$, relativement à $\mathbf{k} / \mathbf{k}_{0}$, capitulent dans le corps des genres $\left(\mathbf{k} / \mathbf{k}_{0}\right)^{(*)}$ de $\mathbf{k}$ (c'est l'extension maximale non ramifiée pour tous les idéaux premiers, finis et infinis, et qui est abélienne sur $\mathbf{k}_{0}$ ). Ce théorème a été conjecturé par T. Tannaka et démontré par F. Terada (voir [14]).

Le cas où $\mathbf{F} / \mathbf{k}$ est une extension cyclique et $[\mathbf{F}: \mathbf{k}]=p$, un nombre premier, a été traité par Hilbert. Sa réponse est le sujet du théorème 94 qui affirme qu'il y a au moins une classe non triviale dans $\mathbf{k}$ qui capitule dans F. De plus, Hilbert avait trouvé le résultat suivant: Soient $\sigma$ un générateur du groupe de Galois de $\mathbf{F} / \mathbf{k}, N$ la norme de $\mathbf{F} / \mathbf{k}, \mathbf{U}_{0}$ le groupe des unités de $\mathbf{k}, \mathbf{U}$ le groupe des unités de $\mathbf{F}$ et $\mathbf{U}^{*}$ le sous-groupe des unités de $\mathbf{U}$ dont la norme, relative à l'extension $\mathbf{F} / \mathbf{k}$, est égale à 1. Alors le groupe des classes de $\mathbf{k}$ qui capitulent dans $\mathbf{F}$ est isomorphe au groupe quotient $\mathbf{U}^{*} / \mathbf{U}^{1-\sigma}=\mathbf{H}^{1}(\mathbf{U})$, le groupe cohomologique de $\mathbf{U}$ de dimension 1 .

A l'aide de ce théorème et de plusieurs résultats sur les groupes cohomologiques des unités, on montre le théorème suivant:

Théorème 1. Soit $\mathbf{F} / \mathbf{k}$ une extension cyclique de degré un nombre premier, alors le nombre des classes qui capitulent dans $\mathbf{F} / \mathbf{k}$ est égal à

$$
[\mathbf{F}: \mathbf{k}]\left[\mathbf{U}_{0}: N(\mathbf{U})\right]
$$

On trouve une preuve de ce théorème dans [9].

Dans le cas général où $\mathbf{F} / \mathbf{k}$ est une extension abélienne K. Miyake avait conjecturé que le nombre des classes qui capitulent dans $\mathbf{F} / \mathbf{k}$ est un multiple de $[\mathbf{F}: \mathbf{k}]$ (voir [12]). Ceci a été démontré par H. Suzuki (voir [13]).

Plusieurs résultats ont été établis; en particulier on a: Soit $\mathbf{k}$ tel que $C_{\mathbf{k}, 2}$, la 2partie du groupe des classes $C_{\mathbf{k}}$ de $\mathbf{k}$, est isomorphe à $\mathbf{Z} / 2 \mathbf{Z} \times \mathbf{Z} / 2 \mathbf{Z}, \mathbf{k}_{2}^{(2)}$ le 2 -corps de classes de Hilbert de $\mathbf{k}_{2}^{(1)}$ et $G_{2}$ le groupe de Galois de $\mathbf{k}_{2}^{(2)} / \mathbf{k}$. On sait par la théorie des corps de classes que $\operatorname{Gal}\left(\mathbf{k}_{2}^{(1)} / \mathbf{k}\right) \simeq C_{\mathbf{k}, 2}$, par suite $\operatorname{Gal}\left(\mathbf{k}_{2}^{(1)} / \mathbf{k}\right) \simeq \mathbf{Z} / 2 \mathbf{Z} \times \mathbf{Z} / 2 \mathbf{Z}$. Alors $\mathbf{k}_{2}^{(1)}$ contient trois extensions quadratiques de $\mathbf{k}$ dénotées par $\mathbf{K}_{1}, \mathbf{K}_{2}$ et $\mathbf{K}_{3}$. D'après 10] on a:

Théorème 2. Soient $\mathbf{k}$ tel que $C_{\mathbf{k}, 2} \simeq \mathbf{Z} / 2 \mathbf{Z} \times \mathbf{Z} / 2 \mathbf{Z}$ et $G_{2}$ le groupe de Galois de $\mathbf{k}_{2}^{(2)} / \mathbf{k}$; alors on a trois types de capitulation:

type 1: Les quatre classes de $C_{\mathbf{k}, 2}$ capitulent dans chacune des extensions $\mathbf{K}_{i} / \mathbf{k}$, $i=1,2,3$. Ceci est possible si et seulement si $\mathbf{k}_{2}^{(2)}=\mathbf{k}_{2}^{(1)}$.

type 2: Les quatre classes de $C_{\mathbf{k}, 2}$ capitulent toutes seulement dans une extension parmi les trois extensions $\mathbf{K}_{i} / \mathbf{k}, i=1,2$, 3. Dans ce cas le groupe $G_{2}$ est diédral.

type 3: Seulement deux classes capitulent dans chacune des extensions $\mathbf{K}_{i} / \mathbf{k}$, $i=1,2$, 3. Dans ce cas le groupe $G_{2}$ est semidiédral ou quaternionique.

Dans toute la suite on désigne par $p$ et $q$ deux nombres premiers tels que $p \equiv$ $1 \bmod 8, q \equiv-1 \bmod 4$ et $\left(\frac{p}{q}\right)=-1, d=p q, \mathbf{k}=\mathbf{Q}(\sqrt{d}, i), \mathbf{k}_{2}^{(1)}$ le 2-corps de classes de Hilbert de $\mathbf{k}, \mathbf{k}_{2}^{(2)}$ le 2 -corps de classes de Hilbert de $\mathbf{k}_{2}^{(1)}$ et $G_{2}$ le groupe de Galois de $\mathbf{k}_{2}^{(2)} / \mathbf{k}$. On montre que l'indice $Q$ des unités de $\mathbf{k}$ est égal à 2; par suite, d'après [2], on a $C_{\mathbf{k}, 2} \simeq \mathbf{Z} / 2 \mathbf{Z} \times \mathbf{Z} / 2 \mathbf{Z}$. Donc $\mathbf{k}_{2}^{(1)}$ contient trois extensions quadratiques de $\mathbf{k}, \mathbf{K}_{1}, \mathbf{K}_{2}$ et $\mathbf{K}_{3}$. Notre but est l'étude de la capitulation dans les trois extensions $\mathbf{K}_{i} / \mathbf{k}, i=1,2,3$. 


\section{Unités de Certains corps de nombres de degré 8 Sur $\mathbf{Q}$}

Soient $d_{1}$ et $d_{2}$ deux entiers naturels sans facteurs carrés et premiers entre eux, $d_{3}=d_{1} d_{2}, \epsilon_{1}$ (resp. $\left.\epsilon_{2}, \epsilon_{3}\right)$ l'unité fondamentale de $\mathbf{k}_{1}=\mathbf{Q}\left(\sqrt{d_{1}}\right)$ (resp. $\mathbf{k}_{2}=$ $\left.\mathbf{Q}\left(\sqrt{d_{2}}\right), \mathbf{k}_{3}=\mathbf{Q}\left(\sqrt{d_{3}}\right)\right), \mathbf{k}=\mathbf{k}_{3}(i), Q$ l'indice des unités de $\mathbf{k}, \mathbf{K}_{0}=\mathbf{k}_{1} \mathbf{k}_{2}, \mathbf{K}=$ $\mathbf{K}_{0}(i), N_{1}$ (resp. $N_{2}, N_{3}$ ) la norme de $\mathbf{K}_{0} / \mathbf{k}_{1}$ (resp. $\mathbf{K}_{0} / \mathbf{k}_{2}, \mathbf{K}_{0} / \mathbf{k}_{3}$ ) et $\mathbf{E}_{\mathbf{k}}$ (resp. $\mathbf{E}_{\mathbf{K}_{0}}, \mathbf{E}_{\mathbf{K}}$ ) le groupe des unités de $\mathbf{k}$ (resp. $\mathbf{K}_{0}, \mathbf{K}$ ).

On sait d'après [11] qu'un système fondamental d'unités (SFU) de $\mathbf{K}_{0}$ est (à une permutation près) l'un des systèmes suivants:

i) $\left\{\epsilon_{1}, \epsilon_{2}, \epsilon_{3}\right\}$;

ii) $\left\{\epsilon_{1}, \epsilon_{2}, \sqrt{\epsilon_{3}}\right\}\left(N_{2}\left(\epsilon_{3}\right)=1\right)$;

iii) $\left\{\sqrt{\epsilon_{1} \epsilon_{2}}, \epsilon_{2}, \epsilon_{3}\right\}\left(N_{3}\left(\epsilon_{1}\right)=N_{3}\left(\epsilon_{2}\right)=1\right)$;

iv) $\left\{\sqrt{\epsilon_{1} \epsilon_{2}}, \sqrt{\epsilon_{3}}, \epsilon_{2}\right\}\left(N_{2}\left(\epsilon_{1}\right)=N_{3}\left(\epsilon_{2}\right)=N_{2}\left(\epsilon_{3}\right)=1\right)$;

v) $\left\{\sqrt{\epsilon_{1}}, \sqrt{\epsilon_{2}}, \epsilon_{3}\right\}\left(N_{3}\left(\epsilon_{1}\right)=N_{3}\left(\epsilon_{2}\right)=1\right)$

vi) $\left\{\sqrt{\epsilon_{1} \epsilon_{2}}, \sqrt{\epsilon_{2} \epsilon_{3}}, \sqrt{\epsilon_{1} \epsilon_{3}}\right\}\left(N_{2}\left(\epsilon_{3}\right)=N_{3}\left(\epsilon_{j}\right)=1, j=1,2\right)$;

vii) $\left\{\sqrt{\epsilon_{1} \epsilon_{2} \epsilon_{3}}, \epsilon_{2}, \epsilon_{3}\right\}\left(N_{3}\left(\epsilon_{1}\right)=N_{3}\left(\epsilon_{2}\right)=N_{2}\left(\epsilon_{3}\right)= \pm 1\right)$.

D'autre part, d'après [3], on a les résultats suivants:

R1: SFU de $\mathbf{k}=\mathbf{Q}(\sqrt{d}, i)$ où $\mathbf{d}$ est un entier naturel différent de 2 et sans facteurs carrés.

Soit $\epsilon_{0}=s+t \sqrt{d}$ l'unité fondamentale de $\mathbf{Q}(\sqrt{d})$.

i) $\mathrm{Si} \epsilon_{0}$ est de norme -1 , alors $\left\{\epsilon_{0}\right\}$ est un SFU de $\mathbf{Q}(\sqrt{d}, i)(Q=1)$.

ii) Si $\epsilon_{0}$ est de norme 1 , alors $\left\{\sqrt{i \epsilon_{0}}\right\}$ est un SFU de $\mathbf{k}(Q=2)$ si et seulement si $s \pm 1$ est un carré dans $\mathbf{N}$ (i.e. si et seulement si $2 \epsilon_{0}$ est un carré dans $\mathbf{Q}(\sqrt{d})$ ). Dans le cas contraire, $\left\{\epsilon_{0}\right\}$ est un SFU de $\mathbf{k}(Q=1)$.

\section{R2: SFU de K.}

Soient $n$ un entier supérieur ou égal à 2 et $\xi_{n}$ une racine primitive $2^{n}$-ième de l'unité. Alors

$$
\xi_{n}=\frac{1}{2}\left(\mu_{n}+\lambda_{n} i\right)
$$

où

$$
\mu_{n}=\sqrt{2+\mu_{n-1}}, \lambda_{n}=\sqrt{2-\mu_{n-1}}, \mu_{2}=0, \lambda_{2}=2 \text { et } \mu_{3}=\lambda_{3}=\sqrt{2} .
$$

De plus, on a: Soient $n_{0}$ le plus grand entier tel que $\xi_{n_{0}}$ appartient à $\mathbf{K},\left\{\epsilon_{1}^{\prime}, \epsilon_{2}^{\prime}, \epsilon_{3}^{\prime}\right\}$ un SFU de $\mathbf{K}_{0}$ et $\epsilon$ une unité de $\mathbf{K}_{0}$ telle que $\left(2+\mu_{n_{0}}\right) \epsilon$ est un carré dans $\mathbf{K}_{0}$ (si elle existe). Alors un SFU de $\mathbf{K}$ est l'un des systèmes suivants:

a) $\left\{\epsilon_{1}^{\prime}, \epsilon_{2}^{\prime}, \epsilon_{3}^{\prime}\right\}$ si $\epsilon$ n'existe pas;

b) $\left\{\epsilon_{1}^{\prime}, \epsilon_{2}^{\prime}, \sqrt{\xi_{n_{0}} \epsilon}\right\}$ si $\epsilon$ existe; dans ce cas on a $\epsilon=\epsilon_{1}^{\prime i_{1}} \epsilon_{2}^{\prime i_{2}} \epsilon_{3}^{\prime}$, où $i_{1}, i_{2} \in\{0,1\}$ (à une permutation près).

Lemme 3. Soient $q$ un nombre premier impair congru $\grave{a}-1$ modulo 4 et $\epsilon=$ $x+y \sqrt{q}$ l'unité fondamentale de $\mathbf{Q}(\sqrt{q})$. Alors $x$ est un entier naturel pair, $x \pm 1$ est un carré dans $\mathbf{N}$ et $2 \epsilon$ est un carré dans $\mathbf{Q}(\sqrt{q})$.

Preuve. Comme $q \equiv-1 \bmod 4$, alors $\epsilon=x+y \sqrt{q}$ est tel que $(x, y) \in \mathbf{Z}^{2}$ et $x^{2}-q y^{2}=1$. D'où $(x+1)(x-1)=q y^{2}$. Du fait que $(x+1)-(x-1)=2$, le plus grand commun diviseur de $x+1$ et $x-1$ est un diviseur de 2. Par suite il existe $\left(y_{1}, y_{2}\right) \in \mathbf{Z}^{2}$ tel que

$$
\left\{\begin{array}{l}
x-1=q^{i} 2^{j} y_{1}^{2}, \\
x+1=q^{i^{\prime}} 2^{j} y_{2}^{2},
\end{array} \quad \text { où } i, i^{\prime}, j \in\{0,1\}, i+i^{\prime}=1 \text { et } 2^{j} y_{1} y_{2}=y,\right.
$$




$$
\left(y_{1} \sqrt{q^{i} 2^{j}}+y_{2} \sqrt{q^{i^{\prime}} 2^{j}}\right)^{2}=2 \epsilon \quad \text { et } \quad \sqrt{2 \epsilon}= \pm\left(y_{1} \sqrt{q^{i} 2^{j}}+y_{2} \sqrt{q^{i^{\prime} 2^{j}}}\right) .
$$

Si $x-1$ est pair, alors $j=1$. En multipliant (1) par $\sqrt{2}$ on trouve que $\sqrt{\epsilon}=$ $\pm\left(y_{1} \sqrt{q^{i}}+y_{2} \sqrt{q^{i^{\prime}}}\right) \in \mathbf{Q}(\sqrt{q})$, puisque $i+i^{\prime}=1$. Comme $\epsilon$ est l'unité fondamentale de $\mathbf{Q}(\sqrt{q})$, alors $\sqrt{\epsilon} \notin \mathbf{Q}(\sqrt{q})$, donc on a une contradiction. Par conséquent, $x$ est pair et $x \pm 1$ est un carré dans $\mathbf{N}$. De plus, comme $x-1$ est impair, alors $j=0$ et $2 \epsilon$ est un carré dans $\mathbf{Q}(\sqrt{q})$.

Théorème 4. Soient $p$ et $q$ deux nombres premiers impairs tels que $p \equiv-q \equiv$ $1 \bmod 4, \mathbf{K}_{0}=\mathbf{Q}(\sqrt{p}, \sqrt{q}), \mathbf{K}=\mathbf{K}_{0}(i)$ et $\epsilon_{1}$ (resp. $\epsilon_{2}, \epsilon_{3}$ ) l'unité fondamentale de $\mathbf{Q}(\sqrt{p})$ (resp. $\mathbf{Q}(\sqrt{q}), \mathbf{Q}(\sqrt{p q})$ ). On suppose que $2 \epsilon_{3}$ est un carré dans $\mathbf{Q}(\sqrt{p q})$. Alors un $S F U$ de $\mathbf{K}_{0}$ est $\left\{\epsilon_{1}, \epsilon_{2}, \sqrt{\epsilon_{2} \epsilon_{3}}\right\}$ et un $S F U$ de $\mathbf{K}$ est $\left\{\epsilon_{1}, \sqrt{i \epsilon_{2}}, \sqrt{\epsilon_{2} \epsilon_{3}}\right\}$.

Preuve. On sait, d'après le lemme 3 , que $2 \epsilon_{2}$ est un carré dans $\mathbf{Q}(\sqrt{q})$. Comme $2 \epsilon_{3}$ est un carré dans $\mathbf{Q}(\sqrt{p q})$, alors $\sqrt{\epsilon_{2} \epsilon_{3}}$ appartient à $\mathbf{K}_{0}$. De plus $\sqrt{\epsilon_{2}} \notin \mathbf{K}_{0}$ et $\sqrt{\epsilon_{3}} \notin \mathbf{K}_{0}$. D'où un SFU de $\mathbf{K}_{0}$ est $\left\{\epsilon_{1}, \epsilon_{2}, \sqrt{\epsilon_{2} \epsilon_{3}}\right\}$. D'autre part $\sqrt{2 \epsilon_{2}} \in \mathbf{K}_{0} \Leftrightarrow$ $\sqrt{i \epsilon_{2}} \in \mathbf{K}$. Par suite, d'après le résultat $R 2,\left\{\epsilon_{1}, \sqrt{i \epsilon_{2}}, \sqrt{\epsilon_{2} \epsilon_{3}}\right\}$ est un SFU de $\mathbf{K}$.

Remarque 5. Soient $p$ et $q$ deux nombres premiers impairs tels que $p \equiv 1 \bmod 8$, $q \equiv-1 \bmod 4$ et $\left(\frac{p}{q}\right)=-1$, et $\epsilon_{3}$ l'unité fondamentale de $\mathbf{Q}(\sqrt{p q})$. Alors $2 \epsilon_{3}$ est un carré dans $\mathbf{Q}(\sqrt{p q})$ (c.a.d. $Q=2)$.

Preuve. Soit $\epsilon_{3}=x+y \sqrt{p q}$. Comme $\epsilon_{3}$ est de norme 1 , alors on a $(x-1)(x+1)=$ $p q y^{2}$.

a) Si $2 p q(x-1)$ ou $2(x-1)$ est un carré dans $\mathbf{N}$, alors on a:

- Si $2 p q(x-1)$ est un carré dans $\mathbf{N}$, alors il existe $\left(y_{1}, y_{2}\right) \in \mathbf{Z}^{2}$ tel que

$$
\left\{\begin{array}{l}
x-1=2 p q y_{1}^{2}, \\
x+1=2 y_{2}^{2},
\end{array} \text { et } \quad \sqrt{\epsilon_{3}}=y_{1} \sqrt{p q}+y_{2} \in \mathbf{k}_{3} .\right.
$$

Comme $\epsilon_{3}$ est l'unité fondamentale de $\mathbf{k}_{3}$, alors $\sqrt{\epsilon_{3}} \notin \mathbf{k}_{3}$. Donc ce cas est impossible.

- Il en est de même pour le cas où $2(x-1)$ est un carré dans $\mathbf{N}$.

b) Si $p(x-1)$ ou $2 p(x-1)$ est un carré dans $\mathbf{N}$, alors on a:

- Si $p(x-1)$ est un carré dans $\mathbf{N}$, alors il existe $\left(y_{1}, y_{2}\right) \in \mathbf{Z}^{2}$ tel que

$$
\left\{\begin{array}{l}
x-1=p y_{1}^{2} \\
x+1=q y_{2}^{2} .
\end{array}\right.
$$

Donc on a $-2=p y_{1}^{2}-q y_{2}^{2}$. Ce qui implique que $\left(\frac{p}{q}\right)=\left(\frac{-2}{p}\right)=1$. Ce qui n'est pas possible.

- Si $2 p(x-1)$ est un carré dans $\mathbf{N}$, on obtient la même contradiction.

c) Si $q(x-1)$ ou $2 q(x-1)$ est un carré dans $\mathbf{N}$, alors on a:

- Si $2 q(x-1)$ est un carré dans $\mathbf{N}$, alors il existe $\left(y_{1}, y_{2}\right) \in \mathbf{Z}^{2}$ tel que

$$
\left\{\begin{array}{l}
x-1=2 q y_{1}^{2} \\
x+1=2 p y_{2}^{2} .
\end{array}\right.
$$

Donc on a $1=p y_{2}^{2}-q y_{1}^{2}$. Ce qui implique que $\left(\frac{p}{q}\right)=1$. Ce qui n'est pas possible.

- Si $q(x-1)$ est un carré dans $\mathbf{N}$, on obtient la même contradiction.

Ainsi donc $p q(x-1)$ ou $(x-1)$ est un carré dans $\mathbf{N}$; ce qui est équivalent à dire que $2 \epsilon_{3}$ est un carré dans $\mathbf{Q}(\sqrt{p q})$ (c.a.d. $\left.Q=2\right)$. 


\section{Capitulation dans Certaines extensions quadratiques De $\mathbf{k}$}

Soient $p$ et $q$ deux nombres premiers, $d=p q, \mathbf{k}=\mathbf{Q}(\sqrt{d}, i)$ et $Q$ l'indice des unités de k. Dans toute la suite on supposera que $p \equiv 1 \bmod 8, q \equiv-1 \bmod 4$ et $\left(\frac{p}{q}\right)=-1$. Comme $Q=2$, alors d'après [2], la 2-partie $\mathbf{C}_{\mathbf{k}, 2}$ du groupe de classes de $\mathbf{k}$ est isomorphe à $\mathbf{Z} / 2 \mathbf{Z} \times \mathbf{Z} / 2 \mathbf{Z}$. Soient $\mathbf{k}_{2}^{(1)}$ le 2 -corps de classes de $\mathbf{k}$ et $\mathbf{k}^{(*)}$ le corps des genres de $\mathbf{k}$ (c'est l'extension maximale non ramifiée pour tous les idéaux premiers, finis et infinis, et qui est abélienne sur $\mathbf{Q}$ ). On va déduire de la section précédente quelques résultats concernant le problème de la capitulation.

Le corps des genres de $\mathbf{k}$ est $\mathbf{k}^{(*)}=\mathbf{Q}(\sqrt{p}, \sqrt{q}, i)$. Soient $\epsilon_{1}$ (resp. $\left.\epsilon_{2}, \epsilon_{3}\right)$ l'unité fondamentale de $\mathbf{Q}(\sqrt{p})$ (resp. $\mathbf{Q}(\sqrt{q}), \mathbf{Q}(\sqrt{p q})$ ) et $\mathbf{k}_{0}^{(*)}$ le sous-corps réel maximal de $\mathbf{k}^{(*)}$. D'après le théorème 4 , on a les propriétés suivantes:

- Un SFU de $\mathbf{k}_{0}^{(*)}$ est $\left\{\epsilon_{1}, \epsilon_{2}, \sqrt{\epsilon_{2} \epsilon_{3}}\right\}$.

- Un SFU de $\mathbf{k}^{(*)}$ est $\left\{\epsilon_{1}, \sqrt{i \epsilon_{2}}, \sqrt{\epsilon_{2} \epsilon_{3}}\right\}$ et un SFU de $\mathbf{Q}(\sqrt{p q}, i)$ est $\left\{\sqrt{i \epsilon_{3}}\right\}$.

Par suite $N\left(\mathbf{E}_{\mathbf{k}^{(*)}}\right)$ est engendré par $\left\{i, \epsilon_{3}\right\}$ et $\mathbf{E}_{\mathbf{k}}$ est engendré par $\left\{i, \sqrt{i \epsilon_{3}}\right\}$. Donc $N\left(\mathbf{E}_{\mathbf{k}^{(*)}}\right) \neq \mathbf{E}_{\mathbf{k}}$. Il vient donc que toutes les classes de $\mathbf{C}_{\mathbf{k}, 2}$ capitulent dans $\mathbf{k}^{(*)}$. En résumé nous avons:

Théorème 6. Soient $\mathbf{k}=\mathbf{Q}(\sqrt{p q}, i)$ avec $p$ et $q$ deux nombres premiers tels que $q \equiv-1 \bmod 4, p \equiv 1 \bmod 8,\left(\frac{p}{q}\right)=-1, \mathbf{k}^{(*)}=\mathbf{Q}(\sqrt{p}, \sqrt{q}, i)$ le corps des genres de $\mathbf{k}$ et $\mathbf{C}_{\mathbf{k}, 2}$ la 2-partie du groupe des classes au sens large de $\mathbf{k}$. Alors toutes les classes de $\mathbf{C}_{\mathbf{k}, 2}$ capitulent dans $\mathbf{k}^{(*)}$.

Théorème 7. Soient $\mathbf{k}=\mathbf{Q}(\sqrt{p q}, i)$ avec $p$ et $q$ deux premiers tels que $p \equiv 1 \bmod$ $8, q \equiv-1 \bmod 4$ et $\left(\frac{p}{q}\right)=-1, \mathbf{k}^{(*)}=\mathbf{Q}(\sqrt{p}, \sqrt{q}, i)=\mathbf{k}(\sqrt{p})$ le corps des genres de $\mathbf{k}, \mathbf{k}_{2}^{(1)}$ le 2-corps de classes de Hilbert de $\mathbf{k}$ et $\mathbf{k}_{2}^{(2)}$ le 2-corps de classes de Hilbert de $\mathbf{k}_{2}^{(1)}$. Alors on a

$$
\mathbf{k}_{2}^{(2)} \neq \mathbf{k}_{2}^{(1)} \Leftrightarrow 4 \mid h\left(\mathbf{k}^{(*)}\right) \Leftrightarrow p=x^{2}+32 y^{2}
$$

Preuve. D'après le théorème $2, \mathbf{k}_{2}^{(2)}=\mathbf{k}_{2}^{(1)}$ entraîne que toutes les classes de $\mathbf{C}_{\mathbf{k}, 2}$ capitulent dans toute sous-extension quadratique $\mathbf{L} / \mathbf{k}$ de $\mathbf{k}_{2}^{(1)} / \mathbf{k}$, et $\mathbf{k}_{2}^{(2)} \neq \mathbf{k}_{2}^{(1)}$ entraîne que toutes les classes de $\mathbf{C}_{\mathbf{k}, 2}$ capitulent dans au plus une sous-extension $\mathbf{L} / \mathbf{k}$ de $\mathbf{k}_{2}^{(1)} / \mathbf{k}$. Par suite, si dans une sous-extension $\mathbf{L} / \mathbf{k}$ exactement deux classes de $\mathbf{C}_{\mathbf{k}, 2}$ capitulent, alors $\mathbf{k}_{2}^{(2)} \neq \mathbf{k}_{2}^{(1)}$ et il existe une extension quadratique $\mathbf{F}$ de $\mathbf{k}_{2}^{(1)}$ telle que $\mathbf{k}_{2}^{(1)} \subset \mathbf{F} \subset \mathbf{k}_{2}^{(2)}$. Ainsi, comme $\mathbf{k}^{(*)} \neq \mathbf{k}_{2}^{(1)}, \mathbf{k}_{2}^{(2)} \neq \mathbf{k}_{2}^{(1)} \Leftrightarrow 4 \mid h\left(\mathbf{k}^{(*)}\right)$ où $h\left(\mathbf{k}^{(*)}\right)$ est le nombre de classes de $\mathbf{k}^{(*)}$.

On désigne par $h(c)$ le nombre de classes au sens large de $\mathbf{Q}(\sqrt{c})$ et par $\mathbf{E}_{c}$ le groupe des unités de $\mathbf{Q}(\sqrt{c})$. Soient $\mathbf{E}_{\mathbf{k}^{(*)}}$ (resp. $\mathbf{E}_{\mathbf{k}_{0}^{(*)}}$ ) le groupe des unités de $\mathbf{k}^{(*)}\left(\right.$ resp. $\left.\mathbf{k}_{0}^{(*)}=\mathbf{Q}(\sqrt{p}, \sqrt{q})\right), h(\mathbf{L})$ le nombre de classes d'un corps de nombres $\mathbf{L}, Q=\left[\mathbf{E}_{\mathbf{k}}: \mathbf{E}_{-1} \mathbf{E}_{p q} \mathbf{E}_{-p q}\right], Q^{\prime}=\left[\mathbf{E}_{\mathbf{k}^{(*)}}: \mathbf{E}_{-1} \mathbf{E}_{p} \mathbf{E}_{-p} \mathbf{E}_{q} \mathbf{E}_{-q} \mathbf{E}_{p q} \mathbf{E}_{-p q}\right]$ et $\epsilon_{1}$ (resp. $\left.\epsilon_{2}, \epsilon_{3}\right)$ l'unité fondamentale de $\mathbf{Q}(\sqrt{p})$ (resp. $\left.\mathbf{Q}(\sqrt{q}), \mathbf{Q}(\sqrt{p q})\right)$. Alors d'après [15] on a

$$
\begin{gathered}
h\left(\mathbf{k}^{(*)}\right)=\frac{1}{2^{5}} Q^{\prime} h(p) h(q) h(-p) h(-q) h(p q) h(-p q), \\
h(\mathbf{k})=\frac{1}{2} Q h(p q) h(-p q) .
\end{gathered}
$$


Comme la 2-partie de $h(\mathbf{k})$ est égale à 4 et $Q=2$, alors la 2-partie de $h(p q) h(-p q)$ est égale à 4 . D'autre part, comme $p \equiv 1 \bmod 8$, alors la 2 -partie de $h(-p)$ est au moins divisible par 4 . Les nombres de classes $h(p), h(q)$ et $h(-q)$ sont impairs. Par suite, on a:

$$
4\left|h\left(\mathbf{k}^{(*)}\right) \Leftrightarrow 2^{5}\right| h(-p) Q^{\prime}
$$

On sait que $\left\{\epsilon_{1}, \sqrt{i \epsilon_{2}}, \sqrt{\epsilon_{2} \epsilon_{3}}\right\}$ est un SFU de $\mathbf{k}^{(*)}$. Les groupes des unités $\mathbf{E}_{-p}, \mathbf{E}_{-q}$ et $\mathbf{E}_{-p q}$ sont réduits à $\{-1,1\}$. Donc $Q^{\prime}$ est l'indice du sous-groupe engendré par $\left\{i, \epsilon_{1}, \epsilon_{2}, \epsilon_{3}\right\}$ dans $\mathbf{E}_{\mathbf{k}^{(*)}}$. Ainsi on a $Q^{\prime}=4$ et $4 \mid h\left(\mathbf{k}^{(*)} \Leftrightarrow 8 \mid h(-p)\right.$. Or, d'après [6], on a $8 \mid h(-p) \Leftrightarrow p=x^{2}+32 y^{2}$. Par suite $4 \mid h\left(\mathbf{k}^{(*)}\right) \Leftrightarrow p=x^{2}+32 y^{2}$.

Corollaire 8. On garde les hypotèses du théorème précédent. Soit $G_{2}$ le groupe de Galois de $\mathbf{k}_{2}^{(2)} / \mathbf{k}$. Alors le groupe $G_{2}$ est de type $(2,2)$ ou bien diédral d'ordre $2^{m}, m>2$. De plus, $G_{2}$ est diédral si et seulement si $p=n^{2}+32 m^{2}$ avec $n$ et $m$ deux entiers naturels; et dans ces conditions $\mathbf{k}^{(*)}$ est l'unique extension quadratique de $\mathbf{k}$, contenue dans $\mathbf{k}_{2}^{(1)}$, où toutes les classes de $\mathbf{C}_{\mathbf{k}, 2}$ capitulent.

Preuve. C'est une conséquence des théorèmes 2 et 7.

Exemples numériques. - Si $d=17 \times 7$, alors le groupe $G_{2}$ est de type $(2,2)$.

- Si $d=41 \times 7$, alors le groupe $G_{2}$ est diédral.

\section{REFERENCES}

[1] A. Azizi, Sur la capitulation des 2-classes d'idéaux de $\mathbf{Q}(\sqrt{d}, i)$. C. R. Acad. Sci. Paris, t. 325, Série I, p. 127-130, (1997). MR 98d:11131

[2] A. Azizi, Sur le 2-groupe de classes d'idéaux de $\mathbf{Q}(\sqrt{d}, i)$. Rendiconti del circolo matematico di Palermo (2) 48 (1999), 71-92. MR 2000d:11131

[3] A. Azizi, Unités de certains corps de nombres imaginaires et abéliens sur $\mathbf{Q}$. Annales des Sciences Mathématiques du Québec 23( 1999), no. 1, 15-21. MR 2000k:11120

[4] A. Azizi, Capitulation of the 2-ideal classes of $\mathbf{Q}\left(\sqrt{p_{1}}, \sqrt{-p_{2}}\right)$. Lecture notes in pure and applied mathematics, vol. 208, p. 13-19, 1999. MR 2000h:11118

[5] A. Azizi, Capitulation des 2-classes d'idéaux de $\mathbf{Q}(\sqrt{2 p q}, i)$. Acta Arithmetica 94 (2000), p 383 - 399. MR 2001k:11221

[6] P. Barruccand et $\mathrm{H}$. Cohn, Note on primes of type $x^{2}+32 y^{2}$, class number, and residuacity. J. reine angew. Math. 238 (1969), 67-70. MR 40:2641

[7] S. M. Chang and R. Foote, Capitulation in Class Field Extensions of Type (p,p). Can. J. Math. vol 32, No.5, (1980), 1229-1243. MR 82i:12013

[8] H. Cohn, The Explicit Hilbert 2-Cyclic Class Fields of $\mathbf{Q}(\sqrt{-p})$. J. reine angew. Math. 321 (1981), 64-77. MR 82e:12011

[9] F. P. Heider und B. Schmithals, Zur Kapitulation der Idealklassen in unverzweigten primzyklischen Erweiterungen. J. reine angew. Math. 336 (1982), 1-25. MR 84g:12002

[10] H. Kisilevsky, Number Fields with Class Number Congruent to 4 mod 8 and Hilbert's Theorem 94. J. Number Theory 8, (1976), 271-279. MR 54:5188

[11] T. Kubota, Über den bizyklischen biquadratischen Zahlkörper. Nagoya Math. J, 10 (1956), 65-85. MR 18:643e

[12] K. Miyake, Algebraic Investigations of Hilbert's Theorem 94,the Principal Ideal Theorem and Capitulation Problem. Expos. Math. 7 (1989), 289-346. MR 90k:11144

[13] H. Suzuki, A Generalisation of Hilbert's Theorem 94. Nagoya Math. J., vol 121 (1991). MR 92h:11098

[14] F. Terada, A Principal Ideal Theorem in the Genus Fields. Tôhoku Math. J. Second Series, vol. 23, 1971, pp. 697-718. MR 46:5285

[15] H. Wada, On the Class Number and the Unit Group of Certain Algebraic Number Fields. Tokyo U., Fac. of Sc. J., Serie I, 13 (1966), 201-209. MR 35:5414

Département de Mathématiques, Faculté des Sciences, Université Mohammed 1, OujDa, Maroc

E-mail address: azizi@sciences.univ-oujda.ac.ma 